\title{
Exploitation durable du poisson sur le cours inferieur du fleuve Bandama (Côte d'Ivoire) : contraintes et recommandations
}

\author{
Kien Kouassi Brahiman 1 ${ }^{*}$, Kouamé Kouakou Augustin², N'Da Amalan Sylvie ${ }^{2}$, Vanga Adja Ferdinand ${ }^{3}$, \\ Kouamelan Essetchi Paul 2 \\ 1 UFR Sciences Biologiques, Université Peleforo Gon Coulibaly de Korhogo, BP 1328 Korhogo, Côte d'Ivoire. \\ Adresse E-mail : kienkouassibrahima@yahoo.fr \\ 2 Laboratoire d'Hydrobiologie et d'Eco-technologie des eaux, UFR Biosciences, Université Felix Houphouët Boigny, \\ 22 Bp 584 Abidjan 22, Côte d'Ivoire. Addresse E-mail : kkouamea@yahoo.fr, E-mail : sylvienda@yahoo.fr, \\ E-mail : kessetch2012@gmail.com \\ ${ }^{3}$ Université Peleforo Gon Coulibaly de Korhogo, BP 1328 Korhogo, Côte d'Ivoire. \\ Addresse E-mail :af_vanga@yahoo.fr
}

Original submitted in on 14th February 2018. Published online at www.m.elewa.org on $31^{\text {st }}$ May 2018 https://dx.doi.org/10.4314/iab.v125i1.4

\section{RÉSUMÉ}

Objectif : Présenter les contraintes au développement de la filière pêche de poisson sur le fleuve Bandama et proposer des recommandations pour une gestion durable de cette ressource ichtyologique.

Méthodologie et résultats : Un suivi quotidien des activités de pêche sur le fleuve Bandama a été fait. Ainsi des enquêtes à passages répétés et des entretiens visant à relever les difficultés auxquelles sont confrontés les acteurs de la filière pêche ont été réalisées de juillet 2013 à juin 2014. Elles tiennent compte des acteurs vivant directement ou indirectement des retombés de la pêche. Les résultats suivants ont été obtenus: des contraintes liées à la mauvaise organisation des acteurs, au manque de moyens, au non respect des règles de la pêche et d'hygiène et l'obstruction des voies de navigation par l'espèce Echornia crassipes et les roches. Conclusion et application des résultats : Pour le développement de la filière pêche, l'État devra encourager les acteurs pour une gestion participative de la filière, entrevoir une amélioration du système de collecte de données statistiques sur la filière pêche de poisson et des conditions de travail des acteurs.

Une telle initiative constitue une base solide dans le processus d'aménagement des pêcheries continentales en Côte d'Ivoire et dans le monde entier.

Mots clés : Fleuve Bandama ; Développement durable ; Contraintes ; Recommandations ; Côte d'Ivoire.

Sustainable exploitation of fish on the lower Bandama River (Côte d'Ivoire): constraints and recommendations

\section{Abstract}

Objective: To present the constraints to the development of the fish industry on the Bandama River and to propose recommendations for a sustainable management of this aquatic resource.

Methodology and results: Daily monitoring of fishing activities on the Bandama River was done. Thus, repeated surveys and interviews to identify the difficulties faced by fishing industry stakeholders were carried out from July 2013 to June 2014. They took into account those who live directly or indirectly from fishing. The following 
results obtained were: constraints related to the bad organization of the stakeholders, the lack of means, the disregard of the rules of fishing and hygiene, the obstruction of the navigation ways by the species Echornia crassipes and the rocks.

Conclusion and application of the results: For the development of the fishing sector, the government will have to encourage the stakeholders for a participative management of the sector, to foresee an improvement of the system of collection of statistical data on the fishing activities of fish and working conditions of the stakeholders. Such an initiative provides a solid foundation in the process of managing continental fisheries in Côte d'Ivoire and around the world.

Keywords: Bandama River; Sustainable development; Constraints Recommendations; Ivory Coast.

\section{INTRODUCTION}

La pêche, est l'une des activités qui permettent à l'Homme de subsister. Elle reste une source appréciable d'approvisionnement en protéines au niveau des fleuves. Cette activité permet également de créer un nombre important d'emplois dans la transformation et la commercialisation du poisson (Kien et al., 2017). Cependant, les ressources aquatiques continentales sont affectées par les activités humaines résultant de constructions, de modifications ou de destructions d'habitats, des écosystèmes et par diverses sources de surexploitation (Dejoux, 1978). Cette situation décrite s'applique bien au cours inférieur du fleuve Bandama. En effet, les travaux de Koné (2012) et ceux de Koudou (2012) respectivement sur les lacs

\section{MATÉRIEL ET MÉTHODES}

Zone d'étude : La zone d'étude est située en aval du lac de Taabo sur le fleuve Bandama. Ces travaux ont été conduits dans trois localités (Fig. 1): Tiassalé $\left(5^{\circ} 53 \mathrm{~N}\right.$ et $\left.4^{\circ} 49 \mathrm{~W}\right)$, N'Zianouan $\left(6^{\circ} 00 \mathrm{~N}\right.$ et $4^{\circ} 49 \mathrm{~W}$ ) et Singrobo $\left(6^{\circ} 05 \mathrm{~N}\right.$ et $\left.4^{\circ} 55 \mathrm{~W}\right)$.

Collecte de données : La présente étude, a permis de réaliser un suivi quotidien des activités de pêche sur le fleuve Bandama. Des enquêtes à passages répétés visant à relever les difficultés auxquelles sont confrontés les acteurs de la filière pêche ont été réalisées de juillet 2013 à juin 2014. Elles tiennent compte des acteurs vivant directement ou indirectement des retombés de la pêche. Le choix des sites d'enquêtes tient compte de leur de Kossou et de Taabo construis sur ce fleuve font état de la surexploitation des stocks de poissons sur ces lacs. Diverses contraintes au développement de la filière crevette sur cette partie du fleuve Bandama ont également été mises en évidence par Mahyao et al.(2013) et Boguhé (2015). Malgré cet état de surexploitation et ces difficultés de la filière pêche, aucun plan de gestion rationnel n'a encore été formulé pour le cours inférieur du fleuve Bandama. Le présent travail, a pour objectif de présenter les contraintes au développement de la filière pêche de poisson sur cette partie du fleuve Bandama, de proposer et de proposer des recommandations pour une gestion durable de cette ressource ichtyologique.

accessibilité et surtout de leur densité démographique et de l'importance de leurs marchés en toute saison. Ces enquêtes consistaient à administrer à chaque pêcheur, un questionnaire et à réaliser des entretiens directs avec les autorités administratives et coutumières locales et les responsables techniques en charge de la gestion de ce plan d'eau pour des informations relatives aux difficultés rencontrées par les acteurs et leurs suggestions pour améliorer leurs conditions de travail et les captures. Cette technique a été complétée par des observations directes sur le terrain pour vérifier les informations reçues des pêcheurs. 


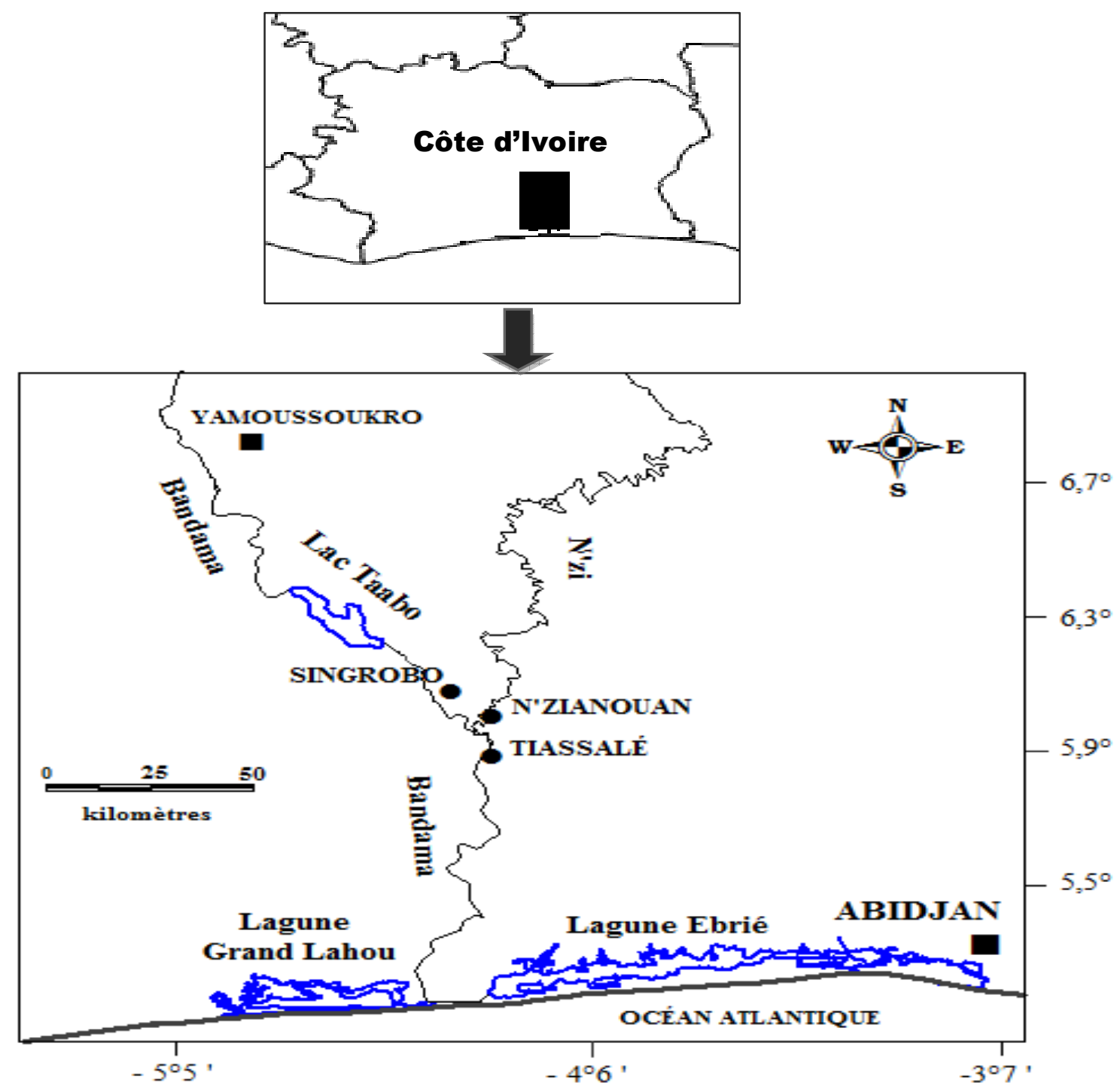

Fig. 1: Situation géographique des stations d'enquêtes $(\bullet)$ sur le cours inférieur du fleuve Bandama (Côte d'Ivoire).

\section{RÉSULTATS}

Organisation de la filière pêche

Au niveau environnemental: Les enquêtes et les différentes observations réalisées au cours de cette étude ont montré que tout le système d'évacuation des eaux usées domestiques provenant des centres urbains, de même que les déchets solides (reste d'aliments, débris végétaux et animaux) des villes et villages environnants aboutissent sans aucun traitement dans le fleuve Bandama. La collecte des déchets se fait de façon informelle dans les différentes localités visitées. II existe également une activité de lessive et de vaisselle permanente sur les roches affleurant dans l'eau. Les usines de transformation d'ananas situées dans la zone d'étude notamment à Tiassalé déversent leurs effluents sans traitement directement dans le fleuve Bandama (Fig. 2). L'extraction industrielle de sable, d'argile et la coupe des arbres pour le bois des ménages, sont très fréquentes dans la zone d'étude. Les grandes bananeraies et pépinières d'hévéa utilisent des engrais et pesticides qui sont drainés vers le fleuve par ruissellement des eaux de pluies ou des canalisations volontaires. La prolifération d'Echornia crassipes qui ont colonisé la surface du fleuve Bandama est également notée dans la présente étude. La présence d'hippopotames sur le cours d'eau menace la sécurité des acteurs. L'ouverture à fréquence élevée des vannes du lac de Taabo en amont de la zone d'étude pendant la 
saison des pluies d'Avril à Juillet correspond à la période de faible production des pêcheurs.

Au niveau de la ressource: Le nombre élevé de pêcheurs (171) dans la zone d'étude a entraîné ces dernières années une diminution des ressources halieutiques et une baisse des revenus des acteurs de la filière.

Prix élevé des équipements et l'accès au crédit: L'activité de pêche nécessite des investissements

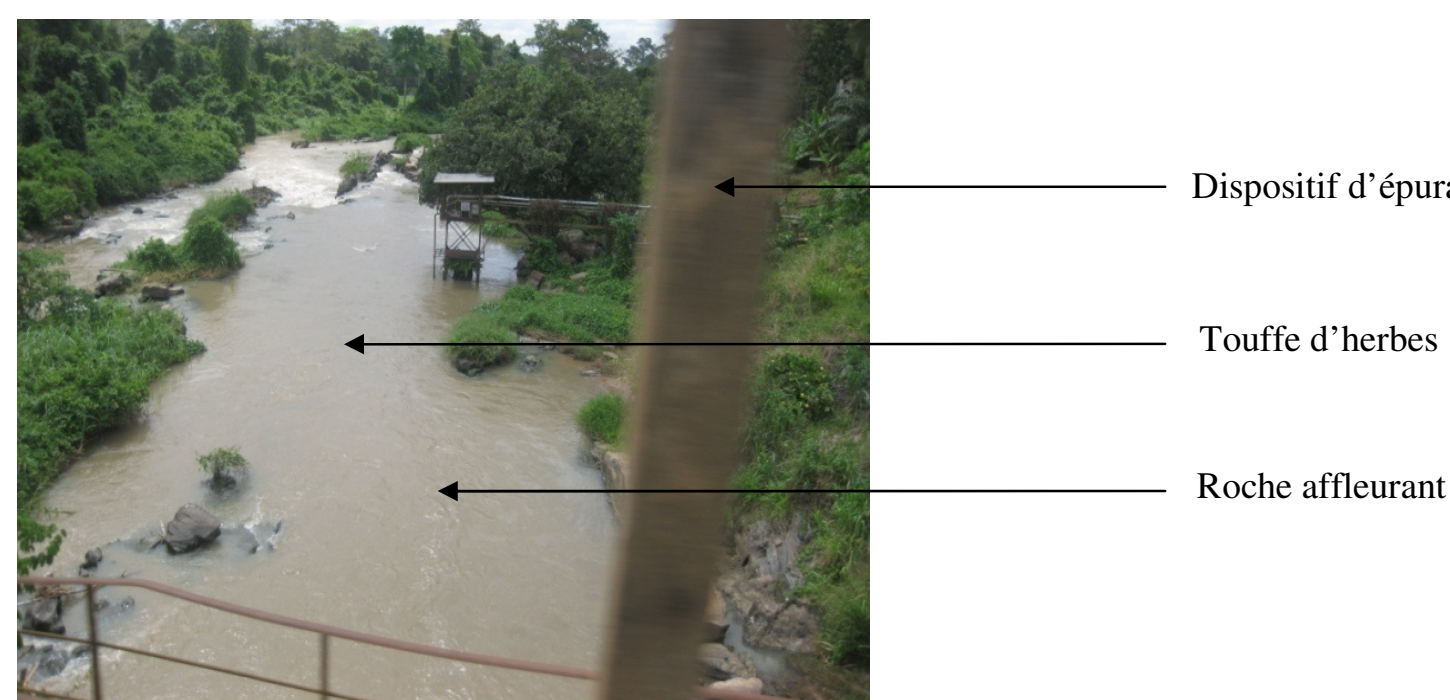

Fig. 2 : Quelques facteurs rendant difficiles la pêche dans la localité de Tiassalé (Bandama, Côte d'Ivoire).

Propriété foncière: Les observations faites dans les différentes localités visitées, montrent que les pêcheurs étrangers n'ont pas accès à certaines zones de pêche considérés comme lieu de rituel par les autochtones. Ainsi, l'accès à ces zones du fleuve est refusé lorsque la demande est faite de façon officielle par ces pêcheurs étrangers. L'accès frauduleux à ces zones est souvent source de conflit entre populations locales et étrangères. Condition de vie: Les pêcheurs vivent généralement dans des conditions précaires, avec des habitations en paille ou en terre (Fig. 3), dans un environnement à hygiène limitée (Fig. 4). L'accès aux soins médicaux et à l'eau potable est limité dans la zone. Le paludisme est importants pour l'achat de pirogue et d'engins de capture. Le prix d'une pirogue peut atteindre 60000 FCFA $(\sim 109$ \$USD) en fonction de la taille et de la qualité du bois utilisé. Les éperviers sont vendus entre 10000 FCFA ( 18 \$USD) et 25000 FCFA ( 45 \$USD). Les prix des filets maillants oscillent entre 20000 et 80000 FCFA ( 36 to 145 \$USD) selon la taille et la qualité du matériel utilisé. présent dans la zone d'étude et certains pêcheurs et infirmiers ont attiré notre attention sur les maladies liées à l'eau. Les réservoirs de prolifération des agents vecteurs (Simulies et Anophèles) de ces maladies, sont fréquents dans les zones visitées. A cela s'ajoute le fait qu'aucune couverture sociale n'existe chez ces acteurs de la pêche. Les acteurs étrangers préfèrent les naturothérapeutes et autres charlatans que de fréquenter les centres de santé. II n'existe aucune forme de coopérative ni d'association entre les acteurs de la filière pêche dans les différentes localités visitées. 


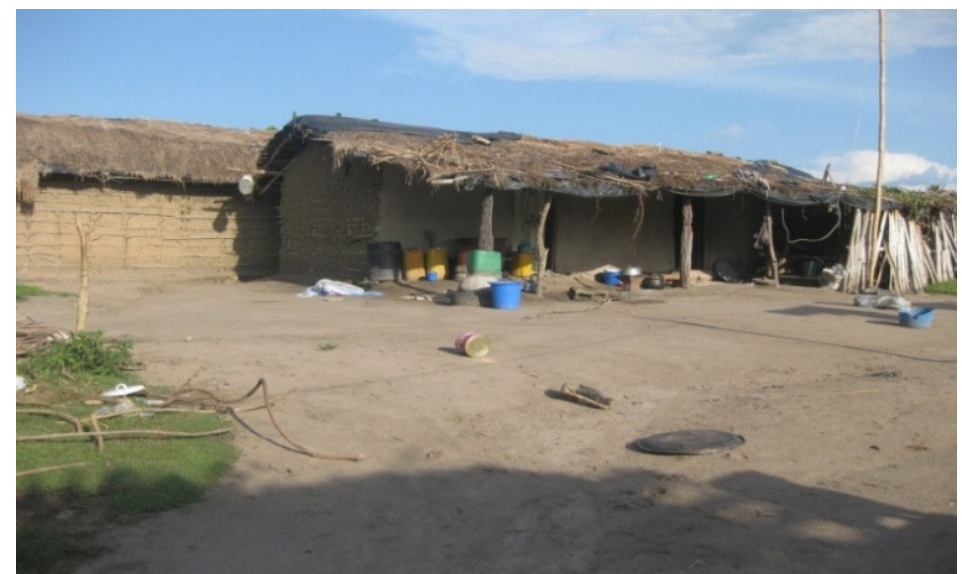

Fig. 3a: Habitation de pêcheurs construite à partir d'argile et de lamelle de palmier à Singrobo.

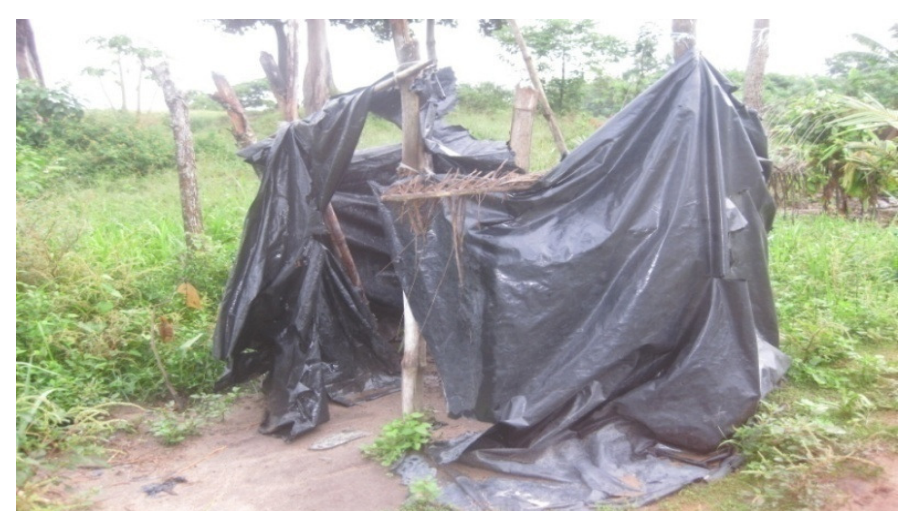

Fig. 3b: Enclos servant de douche et de latrine aux pêcheurs à Singrobo.

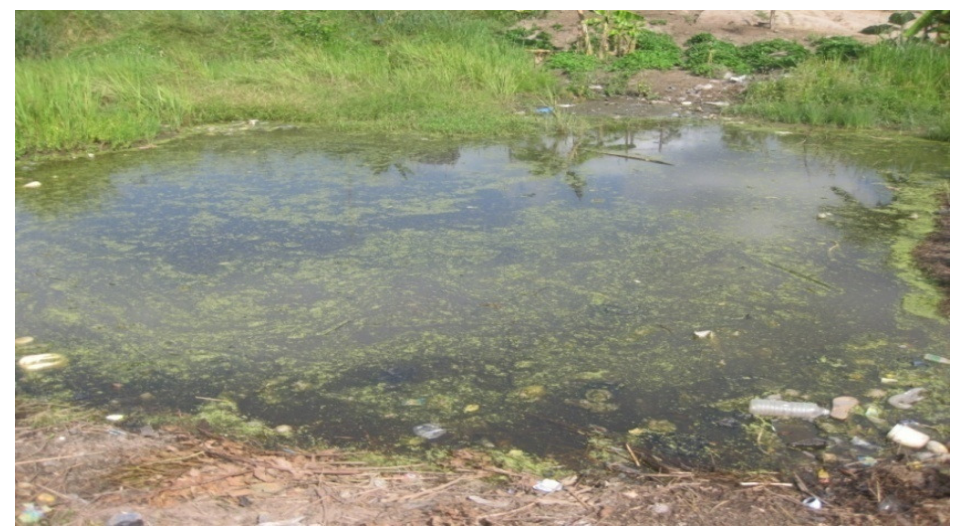

Fig. 4: Eau stagnante favorisant la prolifération d'anophèles femelles, agents vecteurs de paludisme à Singrobo.

Alphabétisation : Dans les localités visitées, la majorité des pêcheurs étrangers est analphabète (61 soit $56 \%$ ) mais certains savent lire et écrire en arabe ( 48 soit $44 \%$ ). Chez ces étrangers, la majorité des enfants en âge d'école ne sont pas scolarisés. Ces enfants sont le plus souvent utilisés pour la pêche en tant qu'aide du parent. Les filles des pêcheurs aident leurs mères dans les ménages au détriment de l'école.
Au niveau de la chaîne de conditionnement et de la vente du poisson. : Cette chaîne est fragilisée par la non utilisation de glace par les pêcheurs et mareyeurs pour des produits facilement périssables. Les grands marchés de débarquement de la pêche artisanale de Tiassalé, de Singrobo et de N'Zianouan sont d'une insalubrité totale car constituent des dépôts de déchets domestiques et des lieux d'aisance des populations riveraines. Les 
poissons sont souvent achetés dans des campements éloignés pour être vendus $1 \mathrm{~h}$ plus tard sans conservation du produit dans de la glace alors que les poissons sont vendus non éviscérés.

Au niveau institutionnel: Le bureau de pêche en charge de la gestion de la pêche sur le fleuve Bandama ne dispose que d'un effectif de trois agents sans matériel informatique ni roulant. Dans les sites visités, il nous a été donné de constater l'inexistence quasi-totale de matériel de travail (engins roulant et ordinateur). Pour le matériel existant, on note une dégradation très avancée au point qu'il ne peut être opérationnel. Les bureaux de pêche des localités investiguées souffrent de sous-effectifs au niveau du personnel et de manque de ressources financières pour couvrir les trois débarcadères officiellement connus à plus forte raison les débarcadères clandestins. II n'existe pas de cadre formel d'échange entre les administrations (Sous-préfet, Directeurs régionaux des ressources animales et halieutiques, du commerce et de l'agriculture) intervenant dans la gestion des ressources halieutiques au niveau local.

Au niveau de la collecte des données. : Les variables qui font l'objet d'évaluation et de suivi par les bureaux de pêche qui sont les premiers producteurs de l'information statistique en matière de pêche sont jugées non exhaustives par la Direction de la Méthodologie du Programme pour les Moyens d'Existence Durables dans la Pêche (MPMEDP). En effet, le travail des agents des bureaux de pêche se limite uniquement au recensement des différents acteurs, à la collecte des différentes taxes, et à la prise des poids des espèces capturées. Des variables telles que le revenu des acteurs, les emplois liés à la pêche, les subventions, et les types d'espèces (poissons d'eau douce, de mer, crevettes, céphalopodes...) ne sont pas renseignées.

Au niveau de la recherche scientifique : Le constat fait sur le terrain montre que tous les projets de recherche sur la gestion des pêcheries sur le fleuve Bandama sont orientés vers les lacs de Taabo et de Kossou. Les investissements orientés sur le cours du fleuve Bandama ont concerné uniquement l'étude de son peuplement (ichtyologique et crevettier). Aucun pisciculteur n'a été recensé dans la zone d'étude. Les volontaires à cet exercice trouvent les infrastructures coûteuses alors qu'ils peuvent exploiter la ressource naturelle déjà à leur portée. A cela s'ajoute l'absence d'encadreurs techniques spécialisés en pisciculture et donc l'impossibilité d'approvisionnement de ces volontaires en alevins et aliments. Aussi est-il qu'il n'existe pas de station d'alevinage dans la zone.
Au niveau de la commercialisation du poisson : Le circuit de commercialisation du poisson sur le cours inférieur du fleuve Bandama est caractérisé par l'insuffisance des infrastructures de base et de son inorganisation. La fixation des prix de vente du produit dépend de la loi de l'offre et de la demande.

Aussi, l'augmentation de la pression fiscale et les difficultés d'acheminement des poissons liés aux conflits sociopolitiques, aux mauvais états de certaines voies ou encore aux procédures administratives non maîtrisées sont parfois contraignantes. On note également un accès difficile aux marchés éloignés des zones de production par le mauvais état des routes.

\section{Contraintes au développement de la filière pêche}

Au niveau environnemental : Le fleuve Bandama est exposé à de très fortes pressions qui détériorent sa qualité. Les déchets liquides tout comme les produits de teinture artisanale des tissus déversés directement dans le fleuve concourent à la pollution de l'eau et donc I'habitat des poissons. Ces produits toxiques détruisent la flore et la faune aquatique. L'extraction de sable et d'argiles favorisent l'érosion du sol mais aussi le dépôt dans l'eau de la matière organique et des sédiments en suspension entrainant une désoxygénation du fleuve Bandama. La prolifération des végétaux aquatiques entrave également avec les arbres et les roches (figure 2) la navigation sur le cours d'eau. Elle peut créer des foyers de reproduction d'anophèles représentant un réel danger pour les pêcheurs. La fréquence élevée de l'ouverture des vannes du barrage de Taabo en saison des pluies entraîne un fort courant d'eau dans le cours inférieur du Bandama, ce qui emporte les filets. Ces lâchers d'eau provoquent également une onde de crue qui disperse les poissons. Cette dispersion régresse la production des pêcheurs.

Au niveau de la ressource: Le nombre élevé de pêcheurs (171) a provoqué une réduction des revenus des pêcheurs. Cette situation les oriente vers des activités complémentaires notamment l'agriculture, la chasse et le commerce. La pêche devient une activité secondaire pour certains d'entre eux.

Prix élevé des équipements et l'accès au crédit : Les prix du matériel de pêche sont jugés élevés par les acteurs. II n'existe pas de coopérative des acteurs dans la zone d'étude. Cette situation rend difficile l'accès au crédit pour le financement de la filière pêche.

Alphabétisation : Le taux élevé de non scolarisés parmi les acteurs de toute nationalité rend difficile la compréhension des textes qui régulent la filière pêche. Cela entraine des incompréhensions qui aboutissent à des conflits. 
Au niveau de la chaîne de conditionnement et de la vente du poisson. : La non utilisation de glace pour la conservation du poisson pêché peut favoriser la dégradation rapide du produit. Cette absence de glace et l'insalubrité des marchés de débarquement ne garantissent pas la bonne qualité du produit de pêche. La fixation aléatoire des prix de vente du poisson ne permet pas un meilleur contrôle des prix sur le marché.

Au niveau institutionnel : Le manque de moyens des services en charge de la gestion de la pêche ne permet pas un contrôle et une surveillance efficace des activités de pêche et une évaluation correcte des données lui afférent. Cette gestion de la ressource halieutique ne peut être efficace sans une coordination entre tous les acteurs du fleuve.

Recommandations : Vu l'intérêt économique que revêt l'activité de pêche sur le fleuve Bandama, des mesures s'imposent pour une exploitation durable de la ressource. Au regard des difficultés énumérées, il faudra donc que l'État:

-Sensibilise les acteurs pour une gestion participative en menant des actions d'information sur les textes législatifs et de sensibilisation sur leur application, sur les droits, les rôles et les devoirs des pêcheurs dans la gestion de la pêche. Cela pourrait rendre plus facile le respect des règles et règlements de la filière pêche.

-Améliore le système de collecte de données sur la filière pêche car l'amélioration de la

situation de la pêche sur le fleuve Bandama passe nécessairement par l'existence de données statistiques fiables sur ce secteur de la pêche. L'État doit donc renforcer le système de collecte déjà en place par des ingénieurs statisticiens spécialisés en pêche pour des données améliorées. L'appui matériel et financier de ces services est plus que nécessaire aujourd'hui.

-Recense les campements et villages, la population et les embarcations des pêcheurs pour évaluer les effectifs et comptabiliser les pirogues. Ce recensement pourrait être effectué chaque année, ce qui permettrait aux gestionnaires de maîtriser le nombre des pêcheurs.

-Redynamise le système de collecte de données sur les captures car sans données initiales

de l'état de la ressource et de l'exploitation, il est difficile de gérer de façon durable la ressource. Ainsi, l'État doit aider les pêcheurs à comptabiliser leur production car les pêcheurs et leurs femmes qui commercialisent le poisson, connaissent les différentes espèces de poissons. Cette technique permettrait à l'État à la fois de limiter les coûts de suivi pour l'administration et d'impliquer les pêcheurs dans la gestion de la ressource. Un agent des bureaux de pêche pourrait alors récupérer ces données une fois par semaine ou tous les mois pour un contrôle en vue de leur validation.

-Recycle les agents des bureaux de pêche aux nouvelles techniques de suivi-évaluation de la filière pêche car ce modèle permettra un contrôle efficace de la ressource depuis sa capture jusqu'à sa consommation. L'État devrait donc assurer aux agents des séminaires de formation sous l'expertise d'un systématicien en Ichtyologie pour l'importance des résultats attendus. II serait donc important de faire des campagnes de sensibilisation sur les conséquences de l'utilisation inappropriée de certains engins ou des mailles plus réduites sur le fleuve. Pour lutter contre la raréfaction des poissons évoquée par les pêcheurs, l'État pourra devra recoloniser des zones fortement exploitées par un aménagement du réseau hydrographique. L'objectif serait de circonscrire des zones interdites à la pêche à certaines périodes notamment pendant la saison des pluies ce qui permettrait un renouvellement de la ressource. Une autre alternative serait d'encourager les acteurs à la pratique de la pisciculture.

-Interdise l'utilisation des mailles non réglementaires (inférieures à $45 \mathrm{~mm}$ ) pour les nasses. Après la phase de sensibilisation, l'État devra imposer des amendes jusqu'à l'interdiction complète de pêcher pour tout individu contrevenant. L'objectif est d'éviter la trop grande proportion des juvéniles dans les captures puisque les nasses sont les engins les plus utilisés sur le cours inférieur du fleuve Bandama.

-Sensibilise les acteurs sur les conditions hygiéniques et sanitaires et la fixation des prix; il est impératif de sensibiliser les acteurs de la filière à l'hygiène, à la qualité des produits, au développement de la chaîne du froid et à la vente du produit au kilogramme. En effet, l'État doit imposer la conservation du produit de pêche dans des caisses isothermes avec de la glace, ce qui permettrait une meilleure conservation du produit. La vente au kilogramme permettra une meilleure estimation des revenus des acteurs et même de la production sur le fleuve. A cet effet, l'État pourra penser à la construction de véritables débarcadères pour mieux contrôler cette activité sur le cours inférieur du Bandama.

- Incite les acteurs de la pêche à la mise en place de coopérative. L'État devrait initier des politiques visant à sensibiliser les différentes communautés de pêcheurs à la mise en place d'une coopérative et à l'épargne pour améliorer leur condition de travail. Cette coopérative pourrait leur permettre de bénéficier des financements de l'État ou des institutions internationales. -Améliore les conditions de vie des acteurs de la pêche; en leur assurant l'accès à l'eau potable et aux soins de santé. 
Les autorités sanitaires doivent sensibiliser les différents acteurs sur l'hygiène en insistant surtout sur les maladies liées à l'eau, notamment le paludisme très fréquent dans la zone. L'État devrait encourager à l'alphabétisation et à la scolarisation des enfants des pécheurs non-Ivoiriens et

\section{DISCUSSION}

Les observations faites sur le cours inférieur du fleuve Bandama ont montré que l'activité de pêche est influencée par les rejets toxiques, la présence des végétaux (Echornia crassipes) sur l'eau et l'ouverture permanente des vannes du barrage de Taabo situé en amont. Nos résultats corroborent avec ceux de Hickling (1961); Kayalto et Mbofung (2009) qui ont montré la présence des polluants d'origine agricole et industrielle respectivement dans le Mékong et dans le lac Tchad. Plusieurs autres travaux en Afrique notamment au Ghana (Biney, 1982) ; au Nigéria (Oluwande, 1983) et au Cameroun (Bopelet, 1983) ont indiqué la présence de rejets résiduaires dans les eaux continentales africaines. Toutes ces situations aboutissent à la pollution, l'eutrophisation, entravent la navigation et la réduction $\mathrm{du}$ taux d'oxygène qui seraient les principales causes de la disparition de certaines espèces dans les captures sur ce plan d'eau et même des personnes. En effet, les métaux lourds issus des polluants peuvent affecter les organismes directement en s'accumulant dans leurs corps et leur bioamplification dans la chaîne alimentaire pourrait entrainer des maladies chez les consommateurs (Unlu et Gumgum, 1993). La FAO (1999) estime à cet effet que la principale menace à la durabilité des ressources halieutiques continentales est moins leur surexploitation que la détérioration de l'environnement. Les résultats de nos enquêtes montre que la gestion dans la zone d'étude s'appui sur un organigramme dont l'examen montre malheureusement le manque de coordination entre les différentes structures qui le compose. Cela expliquerait l'incohérence observée pour une même donnée à différentes sources. Les bureaux de pêche présentent des effectifs, des moyens matériels et financier insuffisants. Les données fournies par ces bureaux ne concernent que les prises de poids des captures, la collecte des taxes et le recensement des acteurs. Ce constat est parfaitement décrit sur le Lac de

\section{CONCLUSION}

A la lumière de notre étude, la pêche continentale en Côte d'Ivoire est un secteur majeur de l'économie nationale qui mérite un meilleur contrôle.

-Sur le cours inférieur du fleuve Bandama, la ressource halieutique est soumise à des pressions anthropiques à définir clairement les modalités d'accès à l'eau considérée par les populations autochtones comme leur propriété foncière. Ceci éviterait les conflits avec les nonIvoiriens.

Taabo par Koudou (2012). Cet auteur affirme que ces services sont dans l'incapacité réelle de fournir des données exhaustives. Par conséquent, la section locale ne fournit que des données statistiques peu fiables car ne mentionnant généralement que celles des acteurs qui lui sont accessibles. Cette situation traduit parfaitement l'insuffisance de moyens financier, matériel et humain alloués aux structures responsables de la collecte de ces données par la Direction des Pêches et de l'Aquaculture. Cette Direction elle-même n'a jamais eu les moyens de sa politique bien que chargée de la mise en œuvre de la politique halieutique nationale (Traoré, 1996). Selon cet auteur, cette situation serait due à une pêche continentale qui n'avait jamais été considérée comme un secteur économique prioritaire pour laquelle il n'était pas nécessaire d'entreprendre des actions de développement. La section locale de pêche s'appui sur des articles, décrets et arrêtés ministériels qui n'ont connu de révision depuis 1996 alors que les contextes environnemental et socio-économique sont en perpétuel changement. A cela s'ajoute l'absence de chambres froides, et d'étals stérilisés. Corlay (1993) en relatant les mêmes faits, estimait que ces points posent surtout le problème de leur qualification en tant que pôles structurants. En effet, La non utilisation de glace pour la conservation du poisson favorise la dégradation rapide du produit qui ne garantit pas la bonne qualité du poisson pêché. Concernant les taxes imposées aux acteurs de la filière, elles ne sont pas reparties entre toutes les administrations comme prévue. Une telle situation est mentionnée à Taabo par Anoh (2007) et Koudou (2012). Ces taxes sont sensées appuyer les finances des administrations comme c'est le cas au Sénégal (Camara, 2008). Malheureusement aux dires des autorités administratives, depuis la réouverture de la pêche, la gestion singulière de la Direction tutélaire serait à la base des incompréhensions récurrentes entre les administrations participantes à la gestion locale.

(pollution, surexploitation) et à des insuffisances dans sa gestion. Les gestionnaires manquent de moyens pour la surveillance de l'eau dont les ressources présentent des signes d'exploitation proche d'un état critique. 
-Seule une gestion concertée entre tous les acteurs du fleuve permettrait de réinstaurer la confiance entre les différents partenaires. Le suivi concerté des activités de

\section{REMERCIEMENTS}

Nous remercions les pêcheurs professionnels opérant sur le cours inferieur du fleuve Bandama pour leur aide et leur

\section{RÉFÉRENCES}

Anoh KP, 2007. Pêche, aquaculture et développement en Côte d'Ivoire. Thèse de Doctorat, Université de Nantes, France, $331 \mathrm{p}$.

Biney CA, 1982. Preliminary survey on the state of pollution of the coastal environment of Ghana. Oceanol. Acta, 4 Suppl. (Vol. Spécial) : 39-43

Boguhé GFDH, 2015. Biologie de la reproduction et exploitation de deux espèces de crevette du genre Macrobrachium: M. macrobrachion (Herklots, 1851) et $M$. vollenhovenii (Herklots, 1857) du fleuve Bandama (Côte d'Ilvoire). Thèse de Doctorat, Université Félix HOUPHOUËTBOIGNY, Abidjan, Côte d'Ivoire, 206 p.

Bopelet M, 1983. Mémorandum sur les sciences de la mer dans la République Unie du Cameroun. Yaoundé, Faculté des Sciences, Université de Yaoundé (mimeo), $27 \mathrm{p}$.

Camara MMB, 2008. Quelle gestion des pêches artisanales en Afrique de l'Ouest? Étude de la complexité de l'espace halieutique en zone littorale sénégalaise. Thèse de Doctorat de troisième cycle. Université Cheikh Anta Diop de Dakar, $337 \mathrm{p}$.

Corlay JP, 1993. Les concepts d'espace halieutique: réflexion de géographes sur les pêches maritimes à partir du cas danois. In: ORSTOM/ IFREMER, ed. Question sur la dynamique de 297 l'exploitation halieutique, Colloques et séminaires, Montpellier, 125-140.

Dejoux C, 1978. Traitements des écosystèmes lotiques tropicaux aux insecticides organophosphorés. Risques pour l'environnement. Verhandlungen der Internationalen Vereinigung furTheoretische und Angewandte Limnologie, 20(4): 2708-13.

FAO, 1999. Indicators for sustainable development of marine capture fisheries. FAO Technical Guidelines for Responsible Fisheries, 8, Rome : $68 \mathrm{p}$.

Hickling CF, 1961. Tropical inland fisheries. London, Longman, $287 \mathrm{p}$.

Kayalto B. and Mbofung C, 2009. Contribution a l'évaluation de la contamination par les metaux pêche et la mise en place de systèmes d'assainissement efficaces de l'eau permettront de sauvegarder la ressource ichtyologique.

coopération. Nous sommes également reconnaissances au Bureau des pêches de Tiassalé pour son assistance.

lourds, de trois espèces de poissons, des sédiments et des eaux du lac Tchad. <hal00825482>. $97 \mathrm{p}$.

Kien KB. Vanga AF. Aboua BRD. Kouamelan EP, 2017. Actors, distribution circuits and marketing of fish on Bandama River (Côte d'Ivoire). African Journal of Marketing Management, 9(2): 8-17.

Koné N, 2012. Étude de la pêche, des paramètres des populations et de la biologie de la reproduction du Clupeidae Pellonula leonensis Boulenger, 1916 dans les lacs de barrages de Kossou et de Taabo (fleuve Bandama, Côte d'Ivoire). Thèse de Doctorat, Université Félix HOUPHOUËTBOIGNY, Abidjan, Côte d'Ivoire, 194 p.

Koudou DA, 2012. La pêche sur le lac de Taabo. Thèse de Doctorat, Université Félix Houphouët-Boigny, Abidjan, Côte d'Ivoire, 389 p.

Mahyao GA. Koffi C. Kouassi NC. Yapi-Gnaoré CV, 2013. Pêche et commercialisation des crevettes d'eau douce du fleuve Bandama en Côte d'Ivoire. Tropicultura, 32(2): 95-102.

Oluwande PA, 1983. Pollution levels in some Nigerian rivers. Water Resource, $17:$ 957-63.

Traoré K, 1996. État des connaissances sur les pêcheries continentales ivoiriennes. Rapport de consultation, Avril 1996. Projet FAO. TCP/ IVC 45-53, $140 \mathrm{p}$.

Unlu E. and Gumgum B, 1993. Concentrations of copper and zinc in fish and sediments from the Tigris river in Turkey.Chemosphere, 26(11): 20552061. 